\title{
Behavior Assessment System for Children, Second Edition
}

National Cancer Institute

\section{Source}

National Cancer Institute. Behavior Assessment System for Children, Second Edition. NCI

Thesaurus. Code C121269.

A comprehensive set of rating scales designed to facilitate the differential diagnosis and classification of a variety of emotional and behavioral disorders of children, aid in the design of treatment plans, and provide insight into an individual's thoughts and feelings. 